\title{
Polyetheretherketone (PEEK) Spacers for Anterior Cervical Fusion: A Retrospective Comparative Effectiveness Clinical Trial
}

\author{
Johannes Lemcke ${ }^{*}$, , Ferass Al-Zain ${ }^{1}$, Ullrich Meier ${ }^{1}$ and Olaf Suess ${ }^{2}$ \\ ${ }^{I}$ Department of Neurosurgery, Unfallkrankenhaus Berlin, Germany \\ ${ }^{2}$ Department of Neurosurgery, Charité-Universitätsmedizin Berlin, Germany and Departement of Neurotraumatology \\ and Spine Surgery, German Red Cross Hospital Westend, Berlin, Germany
}

\begin{abstract}
Background: Anterior cervical decompression and fusion (ACDF) is the standard surgical treatment for radiculopathy and myelopathy. Polyetheretherketone (PEEK) has an elasticity similar to bone and thus appears well suited for use as the implant in ACDF procedures. The aim of this study is to examine the clinical and radiographic outcome of patients treated with standing alone PEEK spacers without bone morphogenic protein (BMP) or plating and to examine the influence of the different design of the two spacers on the rate of subsidence and dislocation.

Methods: This retrospective comparative study reviewed 335 patients treated by ACDF in a specialized urban hospital for radiculopathy or myelopathy due to degenerative pathologies. The Intromed PEEK spacer was used in 181 patients from $3 / 2002$ to $11 / 2004$, and the AMT SHELL spacer was implanted in 154 patients from 4/2004 to 12/2007. The follow-up rate was $100 \%$ at three months post-op and $82.7 \%$ (277 patients) at one year. The patients were assessed with the Japanese Orthopedic Association (JOA) questionnaire and radiographically.

Results: At the one-year follow-up there were 118/277 patients with an excellent clinical outcome on the JOA, 112/277 with a good outcome, 20/277 with a fair outcome, and 27/277 with a poor outcome. Subsidence was observed in 13.3\% of patients with the Intromed spacer vs $8.4 \%$ of the patients with the AMT SHELL. Dislocation of the spacer was observed in 10 of the 181 patients with Intromed spacers but in none of the 154 patients with Shell spacers.

Conclusion: The study demonstrates that ACDF with standing alone PEEK cages leads to excellent and good clinical outcomes. The differences we observed in the subsidence rate between the two spacers were not significant and cannot be related to a single design feature of the spacers.
\end{abstract}

Keywords: Anterior cervical decompression and fusion, ADCF, PEEK spacer, subsidence, dislocation.

\section{INTRODUCTION}

Anterior cervical fusion is a routine surgical procedure for degenerative disc diseases in the cervical spine [1,2]. Today, many options are available, including bone grafts, bone cement, and spacers made of titanium, carbon, and synthetic materials.

Despite continual refinements and new developments in the area of cervical implants, there are today still no consensus recommendations for the choice of the most suitable implant materials. Such a material should have mechanical properties similar to that of bone and should also be highly biocompatible.

Polyetheretherketone (PEEK) is a ductile, crystalline thermoplast with long-term stability. It serves as a matrix material for composite material implants. Previous studies have shown that PEEK demonstrates a high degree of biocompatability and thus is permissible as a material for medical implants. PEEK possesses an EM comparable to that of bone and thus seems quite suitable for use as an

*Address correspondence to this author at the Department of Neurosurgery, Unfallkrankenhaus Berlin, Warener Straße 7, D-12683 Berlin, Germany; Tel: +49 (0)30 5681 3701; Fax: +49 (0)30 5681 3703:

E-mail: johannes.lemcke@ukb.de intervertebral implant. Additionally, PEEK distinguishes itself through radiotransparency, high durability, high chemical and thermal resiliency, and favorable friction and wear properties. Because PEEK cages are radiotransparent, they are produced with embedded titanium pins, in order to enable radiological examination of the cage position.

After experience with other spacers, we have been using PEEK spacers for the past 7 years. The aim of this study was to examine the differences between two cervical PEEK spacers concerning the clinical and radiographic outcomes.

\section{METHODS}

\section{Study Design}

This study is a retrospective comparative effectiveness analysis of two different PEEK spacers we used between 29 January 2002 and 25 October 2007 in patients undergoing anterior cervical diskectomy and fusion (ACDF) for degenerative disc disease.

\section{Patients}

All patients had either cervical myelopathy or cervical radiculopathy with paresthesia, hypesthesia, radicular pareses, or radicular pain. The patients had not improved sufficiently from conservative therapy. On MRI, all patients showed a disc prolapse and/or an osteochondrosis in the 
segment corresponding to the neurological symptoms. The level was specified in some cases by myelography with intrathecal contrast medium and a post-myelo-CT of the cervical spine. The exclusion criteria from the present statistical analysis were trauma or prior surgery on the cervical spine.

\section{Surgery}

All patients underwent ACDF according to the modified Smith-Robinson technique with the Caspar instruments. The segment was identified preoperatively and verified intraoperatively by an image intensifier. The intervertebral space was emptied, and osteophytes were removed with a high speed drill. The cartilage portion of the end plate was removed. The PEEK spacer was placed into the intervertebral space under control of the image intensifier. Nothing was placed inside any of the cages. No patient received plate fixation. Intraoperative fluoroscopy ensured the correct placement of spacers.

\section{Implants}

The Intromed PEEK spacer (Intromed Medizintechnik; Heidesee, Germany) was implanted between 3/2002 and $11 / 2004$ (plus one further case in 8/2006) (Fig. 1A). The AMT SHELL spacer (Advanced Medical Technologies; Nonnweiler, Germany) was implanted between 4/2004 and 11/2007 (Fig. 1B). In the overlapping period of time four instrument containers - two of each brand - were in use. The chosen spacer brand depended to the availability of the containers. The height of the spacer was individually chosen by the surgeon according to the subjective impression of stability after testing with a "dummy spacer".

The underlying rationale for the decision to change from the Intromed PEEK spacer to the AMT SHELL spacer was the hypothesis that the retaining pins of the AMT spacer provide more security against dislocation.

The AMT SHELL cage with a width of $14 \mathrm{~mm}$ has a contact surface area between $109 \mathrm{~mm}^{2}$ (14-mm implant) and $129 \mathrm{~mm}^{2}$ (18-mm implant) [3]. The Intromed PEEK spacer has a contact surface area between $159 \mathrm{~mm}^{2}$ and $189 \mathrm{~mm}^{2}$.

\section{Data Collection}

We examined the patients one day preoperatively, one day postoperatively, three months postoperatively, and one year postoperatively. The clinical symptoms were documented according to the Japanese Orthopedic Association
(JOA score) [4]. We calculated the Recovery rate (RR) according to Hirabayashi et al. [5], in order to make comparisons between patients. Following the suggestion of Yoshida [6], we define a recovery rate (RR) of $75 \%$ or more as an excellent outcome, a RR of $50-74 \%$ as a good outcome, a RR of $20-49 \%$ as a fair outcome, and a RR of less than $20 \%$ as a poor outcome.

In addition to the clinical examination, radiography of the cervical spine in two planes was made at every follow-up. The subsidence of the spacers was assessed by two experienced neuroradiologists looking at the radiography of each patient independently and then comparing their assesments. Penetration of the spacer into the vertebral body by 1 millimeter or more was defined as implant subsidence. Any movement of the cage in sagittal direction exceeding the margin of one of the vertebra of the fused segment was defined as dislocation. The results of the bony fusion were not reviewed systematically by a CT scan. Postoperative CT scans were performed in cases of cage dislocation or signs of instability.

\section{Statistical Analysis}

Descriptive statistics were used to characterize the study sample and summarize the clinical findings. The MannWhitney U-Test and chi-square test were used to compare groups.

In order to assess the independent effect of cage brand, time in the study, and other factors on the clinical outcomes, three forward stepwise regression analyses were performed. The dependent outcome variables were RR post-op, RR $3 \mathrm{M}$, and RR 12M. The independent co-variables for all regression analyses were: sex, age, case number, week of the study, spacer brand, mean spacer height, number of levels, C3/C4, $\mathrm{C} 4 / \mathrm{C} 5, \mathrm{C} 5 / \mathrm{C} 6, \mathrm{C} 6 / \mathrm{C} 7$, and C7/T1. Additionally, a logistic regression analysis was performed with subsidence as the dependent outcome variable and the same independent covariables just mentioned.

A subgroup analysis was performed on all patients operated on between 1 April 2004 and 30 November 2004, as this was the period when both brands of spacers were in use with comparable frequency. The data in this subgroup was not normally distributed according to KolmogorovSmirnov and Shapiro-Wilk tests, so non-parametric tests were used. The patients were compared by brand of spacer, using the Mann-Whitney $\mathrm{U}$ test for RR and chi-square test for subsidence.
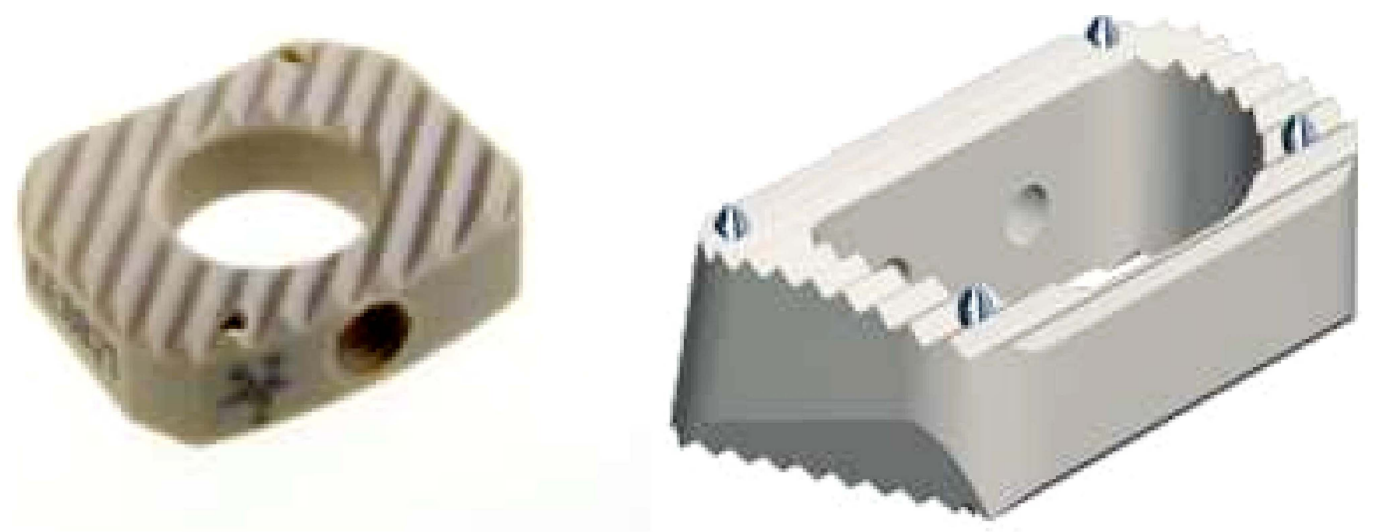

Fig. (1). The Intromed PEEK spacer and the amt SHELL spacer. 


\section{RESULTS}

\section{Study Sample and Implants}

The study sample of 335 consecutive patients contained $179(53.3 \%)$ men. The mean (range) age was $50.6(26-84)$ years. The surgery was single-level in 228 patients $(68 \%)$, two-level in 104 patients (31\%), and three-level in 3 patients (1\%). Surgery was performed at $\mathrm{C} 3 / \mathrm{C} 4$ in 18 patients, at $\mathrm{C} 4 / \mathrm{C} 5$ in 55 patients, at $\mathrm{C} 5 / \mathrm{C} 6$ in 221 patients, at $\mathrm{C} 6 / \mathrm{C} 7$ in 152 patients, and at $\mathrm{C} 7 / \mathrm{T} 1$ in 2 patients.

There were 58 patients who did not have 12 month follow-up data available. Sixteen of these patients were at the end of the study. Thus the ratio of Intermed to SHELL spacers was higher among the completers (156:121) than among the non-completers $(25: 33), p=0.066$. There was also a greater proportion of males among the completers $(56 \%)$ than the non-completers $(43 \%), p=0.083$. Completers had better mean clinical outcomes $(\mathrm{RR})$ than non-completers at post-op (49 vs 43, $\mathrm{p}=0.087$ ) and at 3 month follow-up (64 vs $54, \mathrm{p}=0.01)$. Completers and non-completers did not differ on other variables. Data collection was complete for all other variables at earlier timepoints.

Comparing the patients who received the Intromed vs SHELL spacer, the age distributions appeared comparable (Fig. 2A) and the operated levels were essentially the same (Fig. 2B). The distribution of implant height was consistently $1 \mathrm{~mm}$ less for the AMT SHELL spacer than for the Intromed spacer (Fig. 3).

\section{Complications}

There was no perioperative mortality. We did not observe any superficial wound infections, but one deep infection $(0.3 \%)$ was observed and treated conservatively. Three patients $(0.9 \%)$ showed a transient unilateral paresis of the $\mathrm{N}$. laryngeus recurrens. Two patients $(0.6 \%)$ had paresis of the right $\mathrm{N}$. laryngeus recurrens that persisted at the one year follow-up.

\section{Clinical Outcomes}

Following the categories defined above, directly postoperative $36 / 335$ patients $(10.8 \%)$ had an excellent

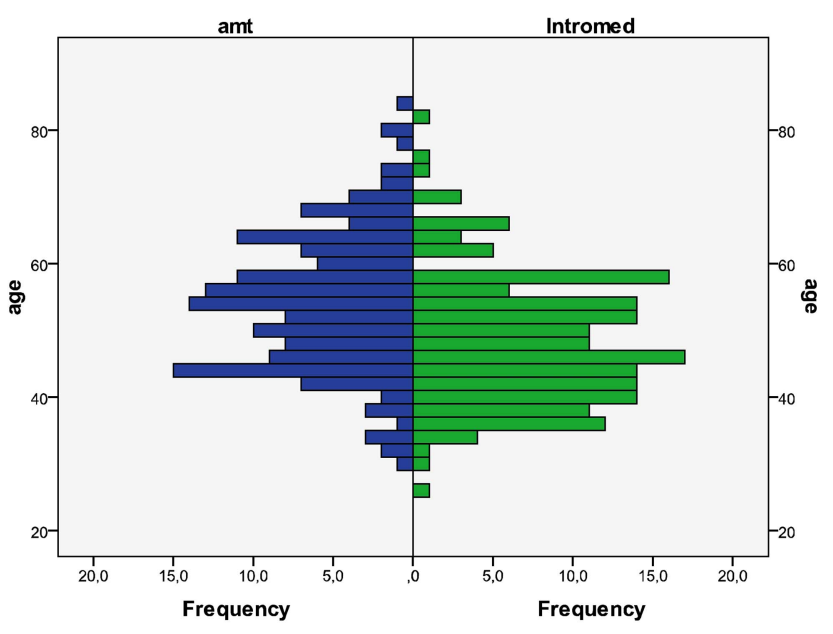

outcome, 180/335 (53.7\%) a good, 80/335 (23.9\%) a fair and $39 / 335$ patients $(11.6 \%)$ a poor outcome. Three months after surgery we found $125 / 335$ patients $(37.3 \%)$ with an excellent outcome, $133 / 335(39.7)$ with a good, $40 / 335(11.9 \%)$ with a fair and $37 / 335(11.1 \%)$ with a poor outcome. At the one year-follow up we registered $118 / 277$ patients $(42.6 \%)$ with an excellent outcome, $112 / 277(40.4 \%)$ with a good, 20/277 $(7.2 \%)$ with a fair and $27 / 277(9.8 \%)$ with a poor outcome.

The mean RR in Interomed vs Shell was 47.2 vs 48.9 at post-op $(\mathrm{p}=0.38), 61.1$ vs 64.0 at 3 months $(\mathrm{p}=0.36)$, and 65.8 vs 69.2 at 12 months $(\mathrm{p}=0.39)$. Thus, there were no significant differences detected in the responder rate between patients with the Intromed $v s$ SHELL spacer (Fig. 4).

\section{Radiographic Outcomes}

Subsidence as defined above was observed in $37 / 335$ patients $(11 \%)$. We did not judge that any of these patients needed surgical revision. Comparing the two types of spacers, there appeared to be a possible difference: $13.3 \%$ of patients with the Intromed spacer $v s 8.4 \%$ of the patients with the AMT SHELL spacer had subsidence $(p=0.16)$ (Fig. 5). There was no difference in the mean clinical outcome (RR 12M) between patients who did vs did not show subsidence (67.5 vs 67.2, $\mathrm{p}=0.94)$.

Dislocation of the spacer was observed in 10 of the 181 patients with 245 Intromed spacers but in none of the 154 patients with 203 Shell spacers, $(p=0.008$ with Yates correction, Fig. 5). There was no statistically significant difference in the mean clinical outcome (RR 12M) between patients who did $v s$ did not have a dislocated spacer (58.5 vs $67.5, \mathrm{p}=0.374)$.

\section{Subgroup Analysis}

There were 40 patients operated between April and November 2004: 18 with Interomed, 22 with SHELL. The sex, age, and number of levels were similar in the two groups. There was no statistically significant difference between the two groups for RR post-op, RR 3M, RR $12 \mathrm{M}$, or subsidence. However, this subgroup analysis was severely underpowered; (e.g. at the measured standardized difference for RR $3 \mathrm{M}$, in this subgroup sample size, the study had a power of $25 \%$ at a p-value of 0.05 , and all others had even

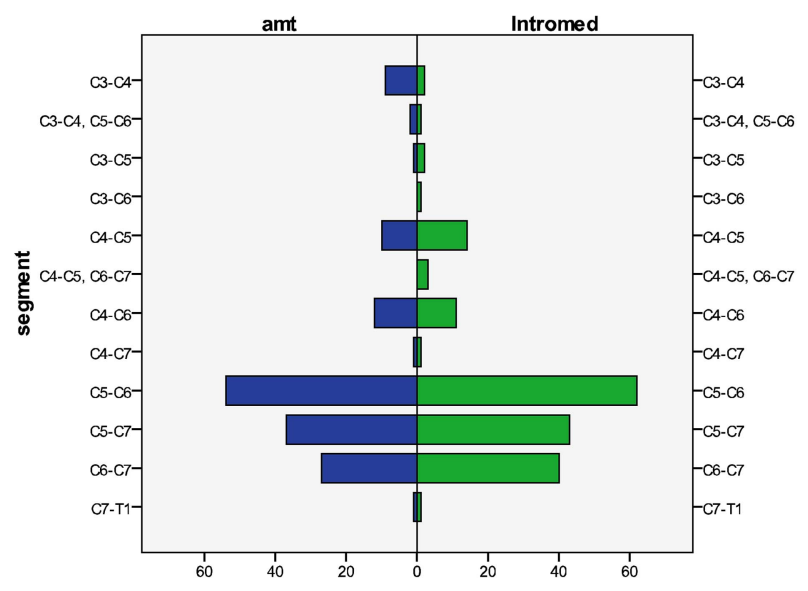

Fig. (2). Histogramms of the age distribution (left) and operated levels (right). 
less power). Thus, no conclusions can be drawn from the subgroup analysis.

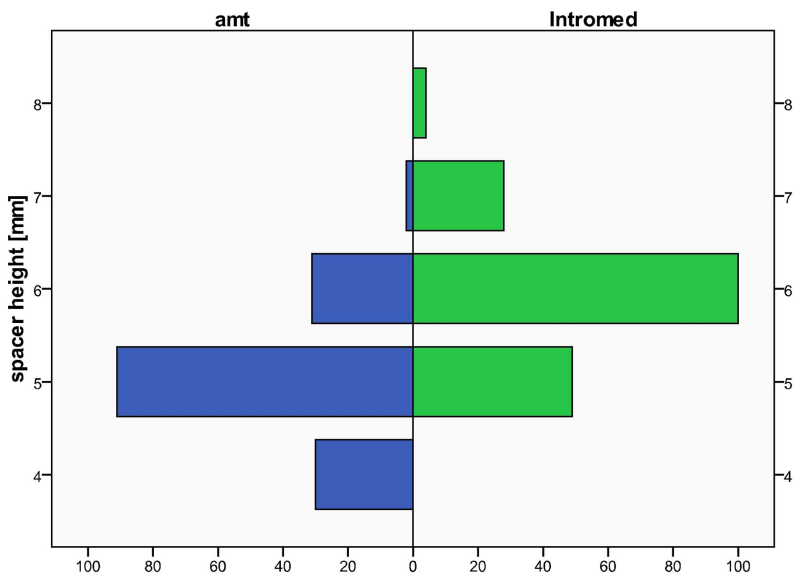

Fig. (3). Histogramm of the heights of the implanted amt spacers (with retaining pins) and of the Intromed spacer (without retaining pins).
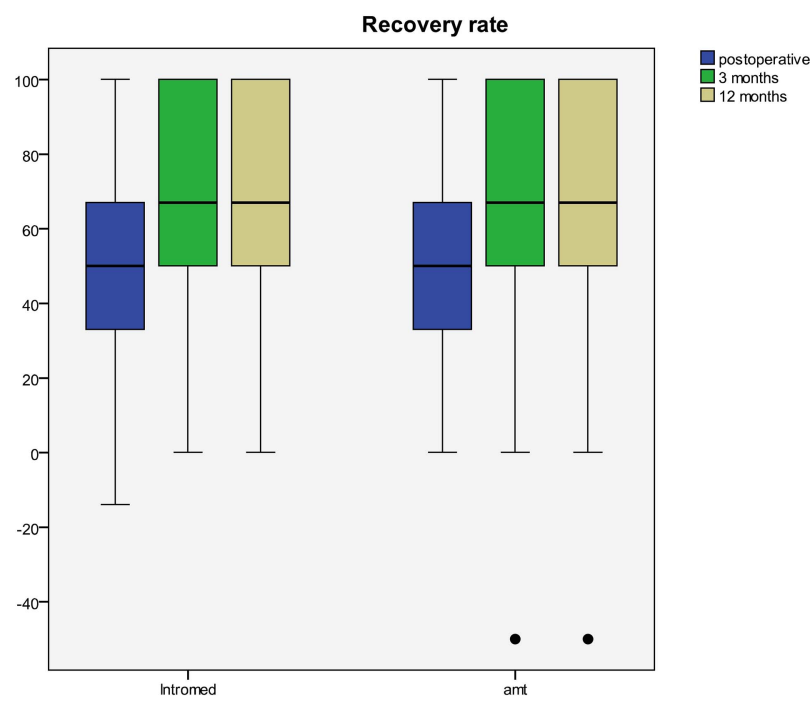

Fig. (4). The Recovery-Rate directly postoperative, after 3 and 12 months for patients with the Intromed and the amt spacer shown as box-and-whisker plots.

\section{Regression Analyses}

The forward stepwise regression identified three factors as significant predictors of $R R$ at post-op: age $(p<0.001)$, operation at $\mathrm{C} 4 / \mathrm{C} 5(\mathrm{p}=0.027)$, and spacer height $(\mathrm{p}=0.034)$. However, this model explained only a very small amount of the variance in the data $\left(\mathrm{R}^{2}=0.056\right.$ (whereby $\mathrm{R}^{2}$ can range from 0 [accounts for none of the data variance] to 1 [accounts for all data variance])), meaning that these factors could barely explain much of the difference in outcomes.

Only age was still a significant predictor for RR $3 \mathrm{M}$ $(\mathrm{p}=0.038)$. But again, this model explained a negligible amount of the data variance $\left(\mathrm{R}^{2}=0.010\right)$. In other words, age had a statistically reliable influence on the 3 month outcomes (such that older patients did worse), but this was a negligible influence in comparison to other factors not captured in the database.

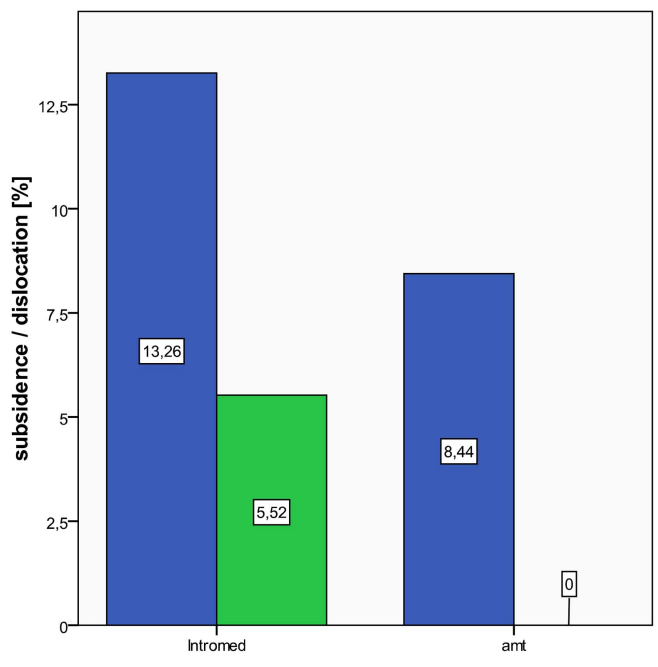

Fig. (5). The rates of subsidence and dislocation compared between Intromed spacer and amt spacer.

At 12 months, the only significant predictor of RR was the number of levels operated $(p=0.039)$, but again this model explained a negligible amount of the data variance $\left(\mathrm{R}^{2}=0.012\right)$.

In the logistic regression analysis, the only significant predictor of subsidence was age $(\mathrm{p}=0.009)$. However age had minimal influence on whether or not subsidence occurred $(\mathrm{OR}=1.05, \quad 95 \% \mathrm{CI}$ of $\mathrm{OR}=1.01-1.09)$. Spacer brand (Intromed) was implicated as a predictor of subsidence but the results were far from statistically significant $(\mathrm{OR}=2.14$, $95 \% \mathrm{CI}$ of $\mathrm{OR}=0.55-8.31, \mathrm{p}=0.27$ ). Case number and week of study were devoid of influence or statistical significance (case number: $\mathrm{OR}=0.99, \mathrm{p}=0.98$; week: $\mathrm{OR}=1.00, \mathrm{p}=0.99$ ); therefore, experience and timeperiod were absolutely not confounding factors for subsidence. Spacer height was also not a significant predictor or subsidence $(\mathrm{OR}=1.19, \mathrm{p}=0.52)$.

\section{DISCUSSION}

Anterior cervical decompression and fusion (ACDF) is the standard surgical treatment for radiculopathy and myelopathy. It aims to decompress the affected nerves by restoring the physiological height of the intervertebral space and the initial width of the neuroforamina. Thus, subsidence of the spacer into the end plate of an adjacent vertebra might result in renewed clinical deterioration.

In our study, subsidence occurred in 39 of the 277 patients with full one-year follow-up (14\%). This is somewhat higher than two previous studies $3.8 \%$ in Hwang et al. [7] and 9\% in Lin et al. [8]), and somewhat lower than in two other studies (19\% in Niu et al. [9] and $33 \%$ in Zevgarides et al. [10]). It seemed that the earlier patients implanted with the Intromed spacer had a higher rate of subsidence than did the more recent patients implanted with the AMT SHELL spacer, though the statistical significance was unreliably weak. The regression analysis proved that experience and timeperiod were not confounding factors. Although the regression analysis implicated the Intromed brand as more prone to subsidence, this finding was not statistically reliable. 
Factors unrelated to the implants themselves could be responsible for the occurrence of subsidence and dislocation. Several studies have asserted that the preparation of the endplates has an influence on subsidence [7, 11-13]. And at least one study has stated that excessive distraction of the intervertebral space has an influence on the probability of subsidence $[14,15]$. In our study, all patients were operated on by the same surgical team, which would argue against the hypothesis that differences in endplate preparation or distraction were responsible for the differences in the rates of subsidence and dislocation.

Alternately, several aspects of the design of the spacers could conceivably explain the difference in their rates of subsidence and dislocation. First, although both spacers were made of PEEK, the titanium pins of the AMT SHELL spacer could have somehow contributed to preventing subsidence.

Second, subsidence could also be related to the amount of contact surface area, whereby less surface area means that more force per area is being transmitted by the spacer to the vertebral endplate. If this was so, we would expect the spacer with less surface area to have a higher rate of subsidence, but in fact we observed the opposite. The AMT SHELL spacer has a smaller surface area, due to its larger central fusion hole, but nonetheless it had a lower rate of subsidence. Similarly one would expect a spacer with less surface area to be less able to grip the vertebral endplate and therefore to dislocate more often. Despite this, the Intromed spacer dislocated more often. So although these geometric/mechanical factors may play a role, they do not explain our clinical observations.

Third, the most likely explanation for the difference in subsidence rates is the difference in the spacer heights used, which is indeed due in turn to a design difference, as we will explain in a moment. We found a linear correlation between spacer height on the one hand and the rates of subsidence and dislocation on the other (Fig. 6). The Intromed spacers we used were consistently $1 \mathrm{~mm}$ taller than the AMT SHELL spacers we used (Fig. 3). Thus the higher rate of subsidence and dislocation of the Intromed spacers (Fig. 5) is apparently due most likely to the fact that they were consistently taller than the AMT SHELL spacers. Of course, the reader will first think that the systematic selection of Intromed spacers that were $1 \mathrm{~mm}$ larger than the AMT Shell spacers has nothing to do with the cage design per se, but instead is the human error of our surgical team.

But we believe that a design feature of the Intromed spacer is indeed what leads the surgeons to systematically choose a spacer that is a bit too big. All operating surgeons in this study checked the stability of the implanted spacers intraoperatively when the spacer was already placed in its final position. Then the Caspar retractor was released and the spacer was still fixed in the application instrument. So when the spacer had no pins (Intromed), a slightly taller spacer was required to create the same feeling of stability in the hands of the surgeon. In other words, the existence of pins in the AMT Shell spacer makes it feel stable with $1 \mathrm{~mm}$ less spacer height. The different rates of dislocation $(5.5 \%$ for Intromed vs $0 \%$ AMT SHELL) support this view. We believe that the x-ray pins of the AMT SHELL spacer accomplish the fixation effectively. By contrast, the Intromed spacer only feels stable by using a slightly taller spacer, which then increases the rates of subsidence and dislocation.

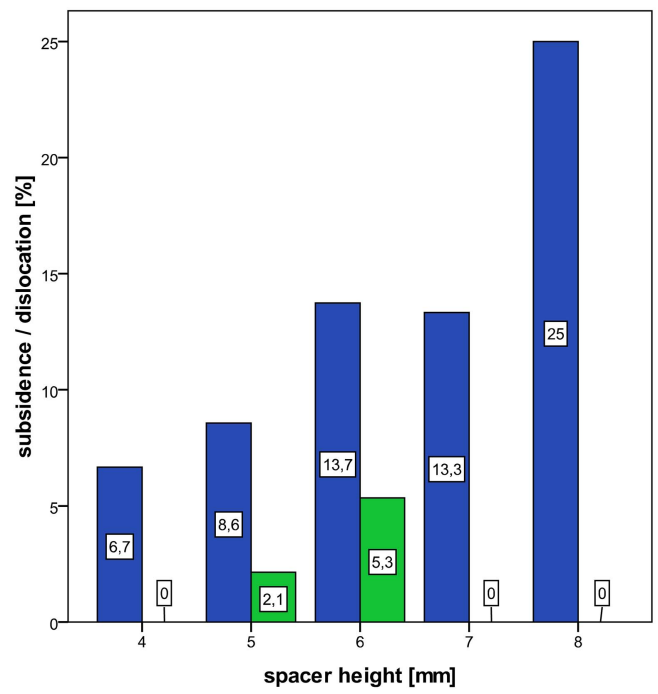

Q subsidence
dislocation

Fig. (6). The rate of subsidence is drawn up against the height of the spacers.

Concluding, we are not able to quote which of these three effects is responsible for the difference, as the cage types differ on several design features, the alleged motivation of the surgeons to use different spacer heights are not confirmable, and the difference between the two spacers is not statistically significant.

Despite this difference in the rates of subsidence and dislocation, we did not observe any differences in the clinical outcomes between the two groups. This is consistent with several other studies that have also not found any influence of subsidence on the actual clinical improvement [7, 14, 1621]. Furthermore, none of the patients with a subsided or dislocated cage needed to be reoperated for this. Nonetheless, we would still consider the Intromed spacer's higher rates of subsidence and dislocation to be an increased safety risk. Studies with larger sample sizes might indeed find more extreme cases of subsidence or dislocation that do negatively impact on clinical outcomes and/or require reoperation.

In summary, ACDF led to substantial recovery in the majority of patients with cervical radiculopathy or myelopathy. Complications were rare. There were no differences in clinical outcomes between patients receiving the Intromed or AMT SHELL spacer. The AMT SHELL spacer had lower rates of subsidence and dislocation than the Intromed spacer. We believe the reason for this is that the retaining pins of the AMT SHELL spacer make it feel more secure at slightly lower spacer heights, and the use of a lower spacer height leads mechanically to less subsidence and dislocation. Although subsidence and dislocation did not affect the clinical outcomes, it could do so. Thus all other factors being equal, we believe patients are a bit safer with the spacer with retaining pins than with the spacer without.

\section{ACKNOWLEDGEMENTS}

We would like to thank: Martin Schomacher for drafting the Introduction; Ayce Atalay, MD, and Michael Hanna, 
PhD, (both Mercury Medical Research \& Writing) for performing data quality-control and contributing to statistical analysis; and Michael Hanna, $\mathrm{PhD}$, for revising the manuscript. The study was supported by "Deutsche Arthrose Hilfe e.V.".

\section{CONFLICT OF INTEREST}

None declared.

\section{REFERENCES}

[1] Cloward RB. The anterior approach for removal of ruptured cervical disks. J Neurosurg 1958; 15(6): 602-17.

[2] Smith GW, Robinson RA. The treatment of certain cervical-spine disorders by anterior removal of the intervertebral disc and interbody fusion. J Bone Joint Surg Am 1958; 40-A(3): 607-24.

[3] Kast E, Derakhshani S, Bothmann M, Oberle J. Subsidence after anterior cervical inter-body fusion. A randomized prospective clinical trial. Neurosurg Rev 2009; 32(2): 207-14.

[4] Fujiwara A, Kobayashi N, Saiki K, Kitagawa T, Tamai K, Saotome K. Association of the Japanese Orthopaedic Association score with the Oswestry Disability Index, Roland-Morris Disability Questionnaire, and short-form 36. Spine 2003; 28(14): 1601-7.

[5] Hirabayashi K, Miyakawa J, Satomi K, Maruyama T, Wakano K. Operative results and postoperative progression of ossification among patients with ossification of cervical posterior longitudinal ligament. Spine 1981; 6(4): 354-64.

[6] Yoshida M, Otani K, Shibasaki K, Ueda S. Expansive laminoplasty with reattachment of spinous process and extensor musculature for cervical myelopathy. Spine 1992; 17(5): 491-7.

[7] Hwang SL, Hwang YF, Lieu AS, et al. Outcome analyses of interbody titanium cage fusion used in the anterior discectomy for cervical degenerative disc disease. J Spinal Disord Tech 2005; 18(4): 326-31.

[8] Lin CN, Wu YC, Wang NP, Howng SL. Preliminary experience with anterior interbody titanium cage fusion for treatment of cervical disc disease. Kaohsiung J Med Sci 2003; 19(5): 208-16.

[9] Niu CC, Chen LH, Lai PL, Fu TS, Chen WJ. Trapezoidal titanium cage in anterior cervical interbody fusion: a clinical experience. Chang Gung Med J 2005; 28(4): 212-21.
[10] Zevgaridis D, Thome C, Krauss JK. Prospective controlled study of rectangular titanium cage fusion compared with iliac crest autograft fusion in anterior cervical discectomy. Neurosurg Focus 2002; 12(1): E2.

[11] Furderer S, Schollhuber F, Rompe JD, Eysel P. Effect of design and implantation technique on risk of progressive sintering of various cervical vertebrae cages. Orthopade 2002; 31(5): 466-71.

[12] Lim TH, Kwon H, Jeon CH, et al. Effect of endplate conditions and bone mineral density on the compressive strength of the graftendplate interface in anterior cervical spine fusion. Spine 2001; 26(8): 951-6.

[13] Truumees E, Demetropoulos CK, Yang KH, Herkowitz HN Failure of human cervical endplates: a cadaveric experimental model. Spine 2003; 28(19): 2204-8.

[14] Lin CN, Wu YC, Wang NP, Howng SL. Preliminary experience with anterior interbody titanium cage fusion for treatment of cervical disc disease. Kaohsiung J Med Sci 2003; 19(5): 208-16.

[15] Francke EI, Demetropoulos CK, Agabegi SS, Truumees E, Herkowitz HN. Distractive force relative to initial graft compression in an in vivo anterior cervical discectomy and fusion model. Spine (Phila Pa 1976 ) 2010; 35(5): 526-30.

[16] Matge G, Leclercq TA. Rationale for interbody fusion with threaded titanium cages at cervical and lumbar levels. Results on 357 cases. Acta Neurochir (Wien ) 2000; 142(4): 425-33.

[17] Matge G. Cervical cage fusion with 5 different implants: 250 cases. Acta Neurochir (Wien ) 2002; 144(6): 539-49.

[18] Assietti R, Beretta F, Arienta C. Two-level anterior cervical discectomy and cage-assisted fusion without plates. Neurosurg Focus 2002; 12(1): E3.

[19] Salame K, Ouaknine GE, Razon N, Rochkind S. The use of carbon fiber cages in anterior cervical interbody fusion: report of 100 cases. Neurosurg Focus 2002; 12(1): E1.

[20] Moreland DB, Asch HL, Clabeaux DE, et al. Anterior cervical discectomy and fusion with implantable titanium cage: initial impressions, patient outcomes and comparison to fusion with allograft. Spine J 2004; 4(2): 184-91.

[21] Peolsson A, Hedlund R, Vavruch L. Prediction of fusion and importance of radiological variables for the outcome of anterior cervical decompression and fusion. Eur Spine J 2004; 13(3): 22934. 